Turkish Journal of Physics

http: / journals.tubitak.gov.tr/physics /

Research Article
Turk J Phys

(2019) 43: $221-227$

(c) TÜBİTAK

doi:10.3906/fiz-1901-23

\title{
Nonlinear frequency conversion of light inside a microcavity
}

\author{
Emre YÜCE ${ }^{1,2, *}$ (1) \\ ${ }^{1}$ Programmable Photonics Group, Department of Physics, Faculty of Arts and Sciences, \\ Middle East Technical University, Ankara, Turkey \\ ${ }^{2}$ The Center for Solar Energy Research and Applications (GÜNAM), \\ Middle East Technical University, Ankara, Turkey
}

Received: 14.01 .2019

Accepted/Published Online: 21.03.2019

Final Version: 08.04.2019

\begin{abstract}
Changing the color of light in a small mode volume is essential for applications that require on-chip operation. Microcavities are profound structures given their property to confine light in a small mode volume. Here, I numerically investigate the frequency conversion of light in a microcavity and show that the frequency converted light intensity is distinguished from an open medium such as an optical fiber or a nonlinear crystal. I observe that the frequency converted light intensity increases with increased rate of change of the refractive index of the microcavity. Notably, this study shows that the intensity of the frequency converted light is maximized when the duration of the index perturbation is matched to the cavity storage time. The results provide a set of optimum parameters for increasing frequency conversion efficiency inside a microcavity.
\end{abstract}

Key words: Microcavity, frequency conversion, nonlinear optics

\section{Introduction}

Changing the color of light is drawing constant attention in applications such as supercontinuum generation [1], tunable laser sources [2], and spectroscopy [3]. The frequency of light is commonly converted via nonlinear crystals [4] or optical fibers [5]. In optical telecommunication technologies, high bandwidth communication lines also require frequency conversion of light with small footprints [6,7]. Optical microcavities that confine light in small mode volumes provide the required small footprint for controlling the frequency of light on-chip.

Frequency conversion of light inside a microcavity is distinguished from nonlinear frequency conversion in an open medium such as an optical fiber. For instance, the time light spends in an open medium is given by the physical size of the medium. On the other hand, a cavity behaves like a folded medium and thereby increases the interaction time of the light with the matter. Moreover, an open medium sustains a broad range of local density optical states, whereas an optical microcavity comprises an increased local density of optical states within a narrow frequency range [8]. For the reasons above, the frequency conversion of light inside a microcavity should be treated discretely from that in an open medium. To date, there has been no study reporting on the effect of the magnitude and duration of the induced nonlinear index change on frequency conversion of light inside a microcavity.

Here, I numerically investigate color conversion of light inside an optical microcavity at various refractive index change amplitudes and durations. I observe that the color of light changes when the refractive index of the microcavity is switched within a short time period in line with previous theoretical and experimental studies

*Correspondence: eyuce@metu.edu.tr 
YÜCE/Turk J Phys

[5,6,8-10]. As predicted, I observe that the frequency converted light intensity increases with increased rate of change of the refractive index of the microcavity. Surprisingly, I observe that the intensity of the frequency converted light is not maximized at short time scales but peaks when the duration of the index perturbation is matched to the cavity storage time.

\section{The methods and model}

The cavity that is employed in this study is composed of alternating Bragg layers and is a 1D structure as shown in Figure 1. The refractive index of the high-index material is chosen as $\mathrm{n}_{1}=3.51$ and the low-index material as $\mathrm{n}_{2}=2.95$, which are typical values for GaAs and AlAs at telecom wavelengths, respectively. The thickness of quarter layers is set to $91.9 \mathrm{~nm}$ and $108.0 \mathrm{~nm}$ for layers with indices $n_{1}$ and $n_{2}$, respectively. The $\lambda$-layer with a thickness of $363.87 \mathrm{~nm}$ is sandwiched between 7 pairs of top mirror and 19 pairs of bottom mirror. The asymmetric design of the cavity allows more leakage of light from the front mirror and increases output from the front mirror $[11,12]$. As shown in Figure 1, air layers of 1- $\mu \mathrm{m}$ thickness are positioned before and after the cavity. With the arrangements above, the cavity possesses a quality factor of $Q=424$, which corresponds to a storage time of $\tau_{c a v}=288 \mathrm{fs}$ at a resonance frequency of $\omega=7807 \mathrm{~cm}^{-1}$.

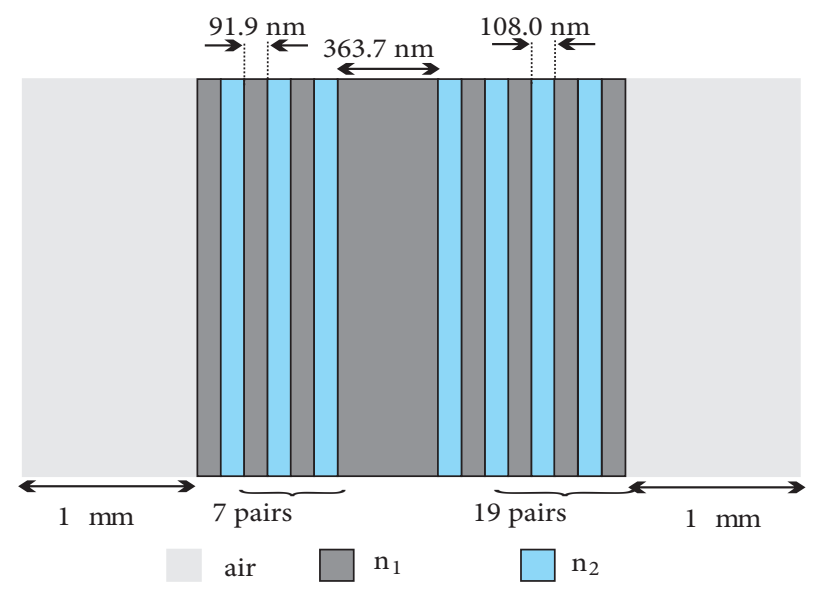

Figure 1. The structure of the cavity and the surrounding media. The $\lambda$-layer with a thickness of $363.7 \mathrm{~nm}$ is sandwiched between 7 and 19 pairs of alternating quarter layers at the top and at the bottom. The thickness of the air layer before and after the structure is $1 \mu \mathrm{m}$.

The time resolved reflected spectra from the microcavity are calculated using the model detailed by Yüce et al. [12]. For this reason, here I provide the necessary steps in our model. An electric field is launched at the structure at $\mathrm{t}_{0}=0$ with a form given in Eq. (1).

$$
E\left(z_{0} t\right)=E_{0}\left(z_{0}\right) e^{-i \omega t} e^{-\left(t-t_{0} / \tau\right)}
$$

where $E_{0}$ is the amplitude of the incident field, $\omega$ the angular frequency, $t$ the time, $z$ the position, and $\tau$ the duration of the Gaussian pulse that is launched at $t_{0}$. The field propagates through the structure for which the refractive index of the $\mathrm{n}_{1}$ layers is set to be time-dependent and the index of the $\mathrm{n}_{2}$ layers and the air layers is invariant during the calculation. For this reason, when the field is within the $\mathrm{n}_{1}$ layers the electric field is reexpressed as in Eq. (2) using the relation $t=n(t) \cdot z-z_{0} / c$.

$$
E(z, t)=E_{0}\left(z_{0}\right) e^{-i \omega\left(n(t) \cdot z-z_{0} / c\right)} e^{-\left(\left(n(t) \cdot z-z_{0} / c\right)-t_{0} / \tau\right)} .
$$


YÜCE/Turk J Phys

When the electric field impinges on an interface a certain portion of the electric field is transmitted and the remaining portion is reflected, providing the Fresnel coefficients. Here the Fresnel coefficients are taken into account in order to calculate the reflected and transmitted ratios, giving the amplitude of forward and backward propagating electric fields at a given position. As a result, the electric field at position $\mathrm{z}$ is expressed as the sum $\left(\mathbf{E}_{\text {sum }}\right)$ of the forward and backward propagating electric field, given in Eq. (3).

$$
E_{\text {sum }}=E^{f}\left(z_{0}, n(z, t) \cdot\left(z-z_{0}\right) / c\right) \cdot t+E^{b}\left(z_{0}, n(z, t) \cdot\left(z-z_{0}\right) / c\right) \cdot r,
$$

where $t$ and $r$ are the Fresnel reflection and transmission coefficients from the relevant interfaces, and $E^{f}$ the amplitude of the forward and $E^{b}$ the amplitude of the backward propagating field. The resultant spectrum is calculated using Eq. (4) at a given position.

$$
\left|E_{\text {sum }}(z, \omega)\right|^{2}=\left|\sum_{0}^{t} E_{\text {sum }}(z, t) \cdot e^{-(i 2 \pi \omega \delta t)}\right|^{2} .
$$

\section{Results and discussion}

The unswitched reflection spectra from the cavity structure are calculated using the arrangement given in Figure 1 and the result is provided in Figure 2. As can be seen in Figure 2, the resonance frequency of the unperturbed cavity is centered at $\omega_{\text {res }}=7807.0 \mathrm{~cm}^{-1}\left(\lambda_{\text {res }}=1280.9 \mathrm{~nm}\right)$. The reflectivity at the stop band is 0.999 and is flat within the frequency range provided in Figure 2.

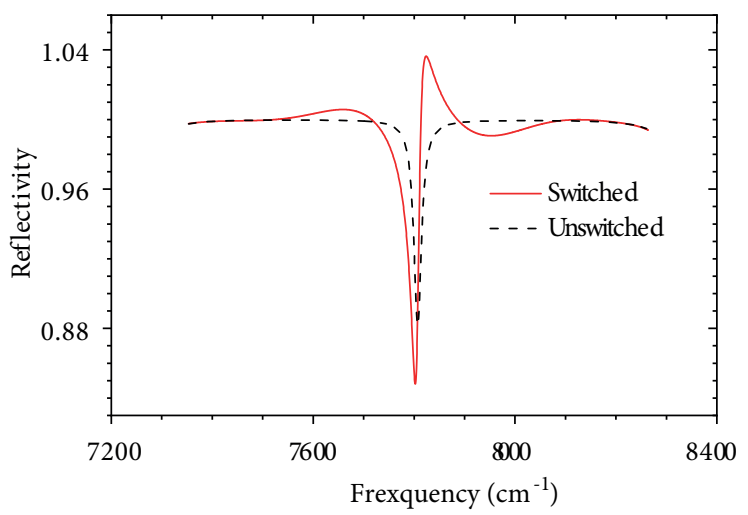

Figure 2. Reflection spectra of a microcavity. The dashed black line shows the spectrum for an unperturbed microcavity and the red solid line shows the spectrum for a switched microcavity.

The refractive index of the cavity is switched via an ultrashort pulse with a duration of $\tau_{p u}=100 \mathrm{fs}$. The refractive index of the $\mathrm{n}_{1}$ layers is assumed to increase linearly via the relation $n=n_{1}+n_{n l} \cdot I_{p u}$, where $n_{1}$ is the unperturbed refractive index, $n_{n l}$ the nonlinear index coefficient, and $I_{p u}$ the intensity of the switch pulse (pump pulse). The switching of the cavity refractive index is considered instantaneous, which is typical for the electronic Kerr effect as previously reported in our experimental research [11]. The nonlinear index coefficient in calculations is taken as $n_{2}=1.5 \times 10^{-5} \mathrm{~cm}^{2} / G W[13]$ and the intensity of the pump pulse is set to $I_{p u}=162 \mathrm{GW} / \mathrm{cm}^{2}$ for the switched spectra presented in Figure 2.

In Figure 2, the resonance frequency of the cavity shifts to a lower frequency $\left(\omega^{\prime}=\left(7802.3 \mathrm{~cm}^{-1}\right)\right.$ due to the increased refractive index. The change in resonance frequency $\left(\omega=4.7 \mathrm{~cm}^{-1}\right)$ corresponds to an index 
change of $\delta n=0.06 \%$. Due to a fast index change within 100 fs the phase of light inside the cavity changes abruptly, which results in frequency change given by Eq. (5) $[5,13]$. In Eq. (5), it can be seen that the rate of change of phase $(\phi)$ results in frequency conversion of light. It should be noted that the phase change is proportional to the change in refractive index $\delta n[5]$.

$$
\delta \omega(t)=-\frac{\partial \phi}{\partial t}
$$

Frequency conversion of light can be observed from the switched spectra presented in Figure 2. When the structure is switched, the reflectivity goes beyond unity, which indicates that there are new frequencies inside the cavity that did not exist before the switch event. The extra light that exceeds unity is a strong indication of frequency converted light but may not provide solid proof. Below I will define solid criteria for determining frequency conversion of light. From the switch spectra it can be deduced that at frequencies lower than the cavity resonance the intensity has decreased and at frequencies higher than the cavity resonance the intensity has increased.

Next, I simulate a pump/probe technique in my calculations in order to provide time resolved spectra at different times. In Figure 3, the resonance frequency of the cavity is plotted as function of time delay between the pump pulse (switch pulse) and the probe pulse. In Figure 3, at zero delay the pump and probe signals arrive at the cavity at the same time. The switching of the cavity refractive index happens within 1 ps as can be seen in Figure 3. Although the duration of the pump pulse is $\tau_{p u}=100 \mathrm{fs}$, the cavity resonance recovers to the unswitched resonance at longer time scales given the storage of the probe field in the cavity, which is $\tau_{c a v}=288 \mathrm{fs}$ [12]. At positive time delays the pump pulse arrives earlier than the probe pulse and at negative time delays the probe pulse arrives earlier than the pump pulse. Depending on the arrival time of the pump pulse the probe field inside the cavity has an increasing or decreasing refractive index. For this reason, the rate of change of phase will be both negative and positive, which results in blue- and red-shifted light.

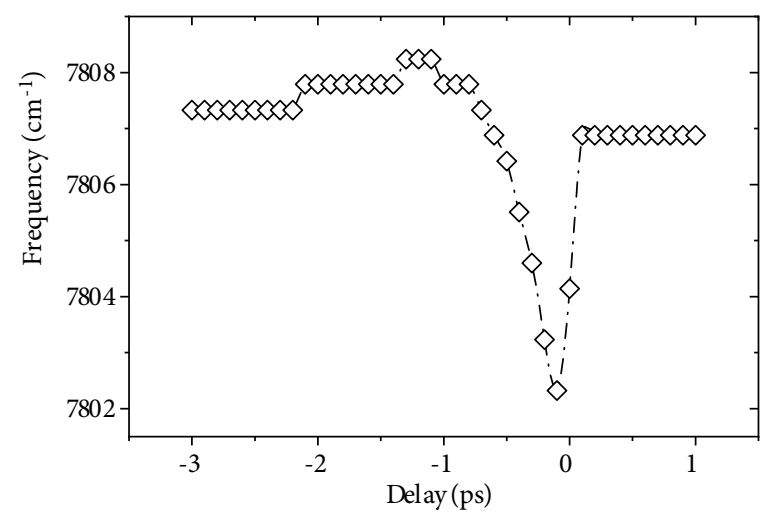

Figure 3. Resonance frequency of the microcavity versus time delay between pump and probe source. At zero delay, the pump and probe signals arrive at the cavity at the same time. At positive time delays, the pump pulse arrives earlier than the probe pulse and at negative time delays the probe pulse arrives earlier than the pump pulse.

Figure 4a gives the reflectivity spectra of the cavity at different time delays between the pump and probe pulse. Indeed, the unswitched spectrum shown in Figure 2 is a cross-section of Figure $4 \mathrm{a}$ at $\delta t=1 \mathrm{ps}$ and the switched spectrum is a cross-section at $\delta t=-0.1 \mathrm{ps}$. As can be seen in Figure $4 \mathrm{a}$, the transient reflectivity goes beyond unity and both the magnitude and frequency of the light exceeding unity change with the arrival time of the pump pulse. 
YÜCE/Turk J Phys
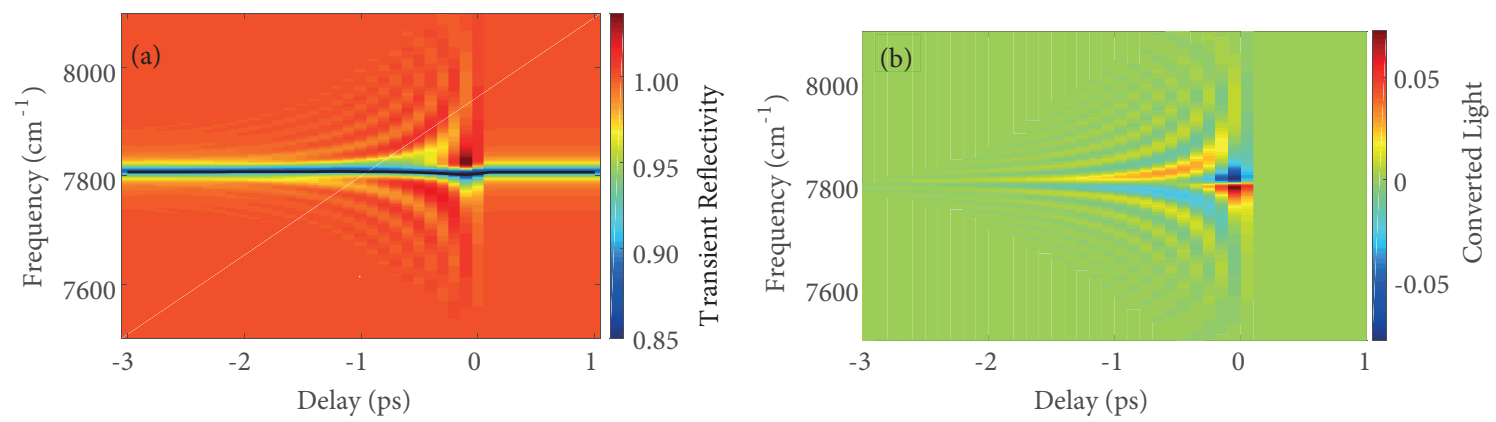

Figure 4. The transient reflectivity of a microcavity at different time delays between the pump and probe pulse. The vertical scale shows the frequency, the horizontal scale the time delay, and the color scale transient reflectivity. (b) Frequency converted light at different time delays. The vertical scale shows the frequency, the horizontal scale the time delay, and the color scale normalized converted light intensity.

Figure 4b shows the normalized intensity of the color converted light, which is calculated by 1-T-R at each time delay. Here $\mathrm{R}$ stands for reflectivity and $\mathrm{T}$ for transmission. Using this definition, I can provide solid proof for color conversion. In the absence of absorption or gain, 1-T-R should be 0 . The values exceeding zero can only be due to new colors that are generated during the switching of the cavity. In Figure 4b at time delays between $\delta t=0$ and $\delta t=0.2 \mathrm{ps}$ the light is red-shifted given the increasing refractive index. At negative time delays beyond $\delta t=0.2 \mathrm{ps}$ the light is mainly blue-shifted. The results are in line with the definition that is provided in Eq. (5). The fringes that are observed at negative time delays are due to interference of light that is reflected from the front face and light that is frequency shifted in the cavity; see Ref. $[8,12,14]$ for details.

Figure 5a shows the color converted light intensity spectra at different pump powers. The time delay between the pump and probe pulse is set to $\delta t=-0.1 \mathrm{ps}$, and the pump pulse duration is set to $\tau_{p u}=100 \mathrm{fs}$ to calculate the spectra in Figure 5. In Figure 5a, it can be seen that the peak intensity of color converted light increases with increasing pump power. Figure 5b shows the integrated color converted light intensity as a function of pump power. The increased intensity of the pump results in increased rate of change of the refractive index and thereby results in increased frequency shift as can be seen in Eq. (5). The results are in agreement with previous predictions and observations [5].

Figure 6a shows the color converted light intensity spectra for different pump pulse durations. As before, the time delay between the pump and probe pulse is set to $\delta t=-0.1 \mathrm{ps}$, and the pump power is set to $I_{p u}=162 \mathrm{GW} / \mathrm{cm}^{2}$ to calculate the spectra in Figure 6. In Figure 6a it can be seen that the peak intensity of color converted light increases with increasing pump pulse duration and the bandwidth gets narrower as expected from Eq. (5). Figure $6 \mathrm{~b}$ shows the integrated color converted light intensity as a function of pump pulse duration. We can see that the total intensity of color converted light increases up to 260 fs and starts to decrease beyond this point. Although the rate of change of phase is increasing with decreasing pump pulse duration, the total intensity of the frequency converted light does not increase. From Figure $6 \mathrm{~b}$ we can see that the maximum amount of color converted light is obtained when the pump pulse duration is matched to the cavity storage time $\left(\tau_{\text {cav }}=288 \mathrm{fs}\right)$. We can conclude that color conversion inside a microcavity shows distinct behavior when compared to that in an open medium.

\section{Conclusion}

The results that focus on nonlinear frequency conversion of light inside a microcavity reveal that frequency converted light intensity inside a microcavity increases with increased rate of change of the refractive index 
YÜCE/Turk J Phys
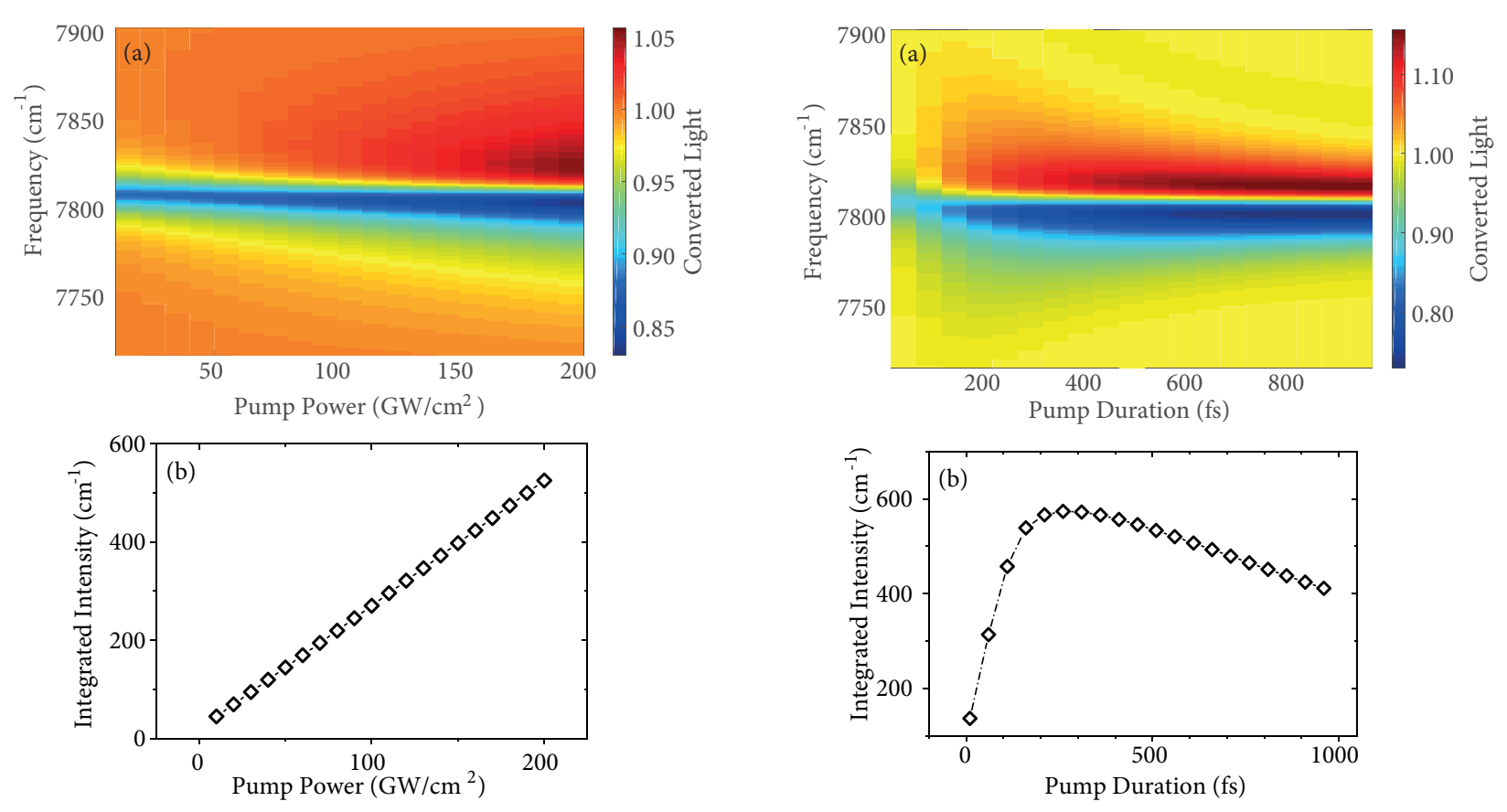

Figure 5. (a) Frequency converted light at different pump powers calculated at time delay $\delta t=-0.1 \mathrm{ps}$. The vertical scale shows the frequency, the horizontal scale the pump power, and the color scale the intensity of color converted light. (b) Integrated intensity of the color converted light as a function of pump power.

Figure 6. (a) Frequency converted light at different pump pulse durations. The vertical scale shows the frequency, the horizontal scale the pump pulse duration, and the color scale the intensity of color converted light. (b) Integrated intensity of the color converted light as a function of pump pulse duration.

of the microcavity. More importantly, this study shows that the intensity of the frequency converted light is maximized when the duration of the index perturbation is matched to the cavity storage time. The results provide optimal parameters for changing the color of light inside a microcavity, which is pursued for on-chip optical telecommunication technologies [15]. Cavities are correspondingly profound structures for controlling quantum light sources that are widely employed in quantum optics. The results will consistently support fundamental studies that aim to control the frequency of single photons [16] using a cavity.

\section{Acknowledgments}

I would like to thank Willem L. Vos and A. Burhan Ünal for useful discussions that support this work.

\section{References}

[1] Dudley, J. M.; Genty, G.; Coen, S. Rev. Mod. Phys. 2006, 78, 1135-1184.

[2] Chang-Hasnain, C. J. IEEE J. Sel. Top. Quant. 2000, 6, 978-987.

[3] Weiss, S. Science 1999, 283 (5408), 1676.

[4] Cerullo, G.; De Silvestri, S. Review of Scientific Instruments 2003, 74, 1-18.

[5] Stolen, R. H.; Lin, C. Phys. Rev. A 1978, 17, 1448-1453.

[6] Preble, S. F.; Xu, Q.; Lipson, M. Nature Photon. 2007, 1, 293-296. 
[7] Kuramochi, E.; Nozaki, K.; Shinya, A.; Takeda, K.; Sato, T.; Matsuo, S.; Taniyama, H.; Sumikura, H.; Notomi, M. Nature Photon. 2014, 8, 474-481.

[8] Yüce, E.; Ctistis, G.; Claudon, J.; Gérard, J. M.; Vos, W. L. arXiv:1406.3586 2016, 1-6.

[9] Notomi, M.; Mitsugi, S. Phys. Rev. A 2006, 73, 051803(R).

[10] Tanabe, T.; Notomi, M.; Taniyama, H.; Kuramochi, E. Phys. Rev. Lett. 2009, 102, 043907.

[11] Yüce, E.; Ctistis, G.; Claudon, J.; Dupuy, E.; Buijs, R. D.; de Ronde, B.; Mosk, A. P.; Gérard, J. M.; Vos, W. L. Opt. Lett. 2013, 38, 374-376.

[12] Yüce, E.; Ctistis, G.; Claudon, J.; Gérard, J. M.; Vos, W. L. Opt. Expr. 2016, 24, 239-253.

[13] Boyd, R. Nonlinear Optics. Academic Press: London, UK, 2008.

[14] Harding, P. J.; Bakker, H. J.; Hartsuiker, A.; Claudon, J.; Mosk, A. P.; Gerard, J. M.; Vos, W. L. J. Opt. Soc. Am. B 2012, 29, A1-A5.

[15] Caspani, L.; Duchesne, D.; Dolgaleva, K.; Wagner, S. J.; Ferrera, M.; Razzari, L.; Pasquazi, A.; Peccianti, M.; Moss, D. J.; Aitchison, J. S.; et al. J. Opt. Soc. Am. B 2011, 28, A67-A82.

[16] Ateş, S.; Agha, I.; Gulinatti, A.; Rech, I,; Rakher, M. T.; Badolato, A.; Srinivasan, K. Phys. Rev. Lett. 2012, 109, 147405. 